\title{
Effects of parenteral administration of enrofloxacin on electrocardiographic parameters in hospitalized dogs
}

\author{
Carlos Fernando Agudelo Ramírez ${ }^{1}$, Peter Scheer ${ }^{2,3}$, Jaroslava Tomenendálová ${ }^{2}$ \\ ${ }^{1}$ University of Veterinary and Pharmaceutical Sciences Brno, Faculty of Veterinary Medicine, Department \\ of Internal Medicine, Clinic of Dog and Cat Diseases, ${ }^{2}$ Faculty of Veterinary Medicine, \\ Department of Physiology, Brno, Czech Republic \\ ${ }^{3}$ St. Anne's Hospital Brno - ICRC, Brno, Czech Republic
}

Received August 10, 2011

Accepted September 19, 2012

\begin{abstract}
The effect of enrofloxacin on the QT interval of the electrocardiogram was studied in 30 hospitalized dogs. The experimental group $(n=15)$ received enrofloxacin parenterally (subcutaneously) at a dose of $5 \mathrm{mg} / \mathrm{kg}$ twice daily and amoxicillin-clavulanate intravenously at a dose of $22 \mathrm{mg} / \mathrm{kg}$ three times daily. The control group $(\mathrm{n}=15)$ received only amoxicillinclavulanate. Electrocardiography was carried out for $5 \mathrm{~min}$ once daily for 6 days. The QT interval was corrected by four different formulae. No differences were found between the two groups or within each group for the duration of the study. On the last day of the study the average QT interval for the control and experimental groups was $213.2 \mathrm{~ms}$ and $202.9 \mathrm{~ms}$, respectively. Enrofloxacin did not cause prolongation of the QT or corrected QT intervals. We can conclude that the parenteral administration of enrofloxacin in non-cardiac dogs does not adversely affect the electrocardiographic indicators (no prolongation of the QT or corrected QT interval) and does not induce ventricular arrhythmias. Parenteral use of enrofloxacin is thus safe and effective in non-cardiac dogs.
\end{abstract}

Quinolone, QT interval, repolarization, canine, ECG

The QT interval (QTi) on the electrocardiogram (ECG) represents the time required to achieve ventricular depolarization and repolarization. This dynamic process depends on the ion channels involved in cardiac repolarization, heart rate (HR), autonomic nervous system activity, age, sex, circadian rhythm, and plasma electrolyte concentrations (mainly potassium and calcium) (Moss 1999; Sheridan 2000). Certain drugs can directly delay the flow of electrolytes within cardiac tissue leading to QT prolongation. Such drugs share the potential to block the cardiac voltage-gated potassium channels, particularly the rapid component (IK $)$ of the delayed rectifier potassium current (Fenichel et al. 2006), which is biophysically and pharmacologically similar in human and dogs (Wang et al. 2003). This effect has been described in antipsychotics, antibiotics, antiarrhythmics, gastrokinetics, anaesthetics and antihistamines, among others (Moss 1999; Dennis et al. 2007; Gupta et al. 2007). Prolongation of the QTi has been demonstrated to represent a risk for fatal cardiac arrhythmia and sudden death in human beings (Moss 1999; Wang et al. 2003).

Several groups of antibiotics such as ampicillin, trimetoprim-sulphonamides, macrolides and quinolones (Table 1) can influence the QTi (Sheridan 2000; Fenichel et al. 2006). Although such adverse effects are known, there are only a few reports in the veterinary literature linking cardiac toxicity and quinolones (Chanoit et al. 2005; Lu et al. 2008; Ghaffari and Parsamehr 2009). Enrofloxacin is a veterinary quinolone bactericidal agent that is metabolized approximately by $10-40 \%$ to ciprofloxacin in the liver (Boothe et al. 2001). In dogs, both enrofloxacin and ciprofloxacin can be found in different tissues including the myocardium $2 \mathrm{~h}$ after parenteral application of enrofloxacin (Boothe et al. 2001). To date, no studies of the effects of enrofloxacin on the ECG in dogs have 
Table 1. Antibacterial drugs related to QTi prolongation.

\begin{tabular}{cl}
\hline Risk category & Antibiotic \\
\hline A & Clarithromycin \\
& Erythromycin \\
& Grepafloxacin \\
Sparfloxacin \\
B & Azithromycin \\
& Gatifloxacin \\
& Gemifloxacin \\
& Moxifloxacin \\
& Telithromycin \\
& Levofloxacin \\
& Ofloxacin \\
& Roxithromycin \\
& Ampicillin \\
& Ciprofloxacin \\
Crimetoprim + Sulphonamides
\end{tabular}

A - strong clinical evidence that provokes TdP, B - isolated reports or insufficient evidence of polymorphic ventricular tachycardia, $\mathrm{C}$ - drugs with questionable reports been reported, although other quinolones have been studied. Recent studies concluded that the ECGs of dogs receiving continual infusion (Ghaffari and Parsamehr 2009) or higher oral (p.o.) doses (5-200 mg/kg) of ciprofloxacin were unchanged (Toyoshima et al. 2005).

The aim of this study was to test the hypothesis that parenteral enrofloxacin can prolong QTi (corrected and uncorrected) on the electrocardiogram in dogs.

\section{Materials and Methods}

\section{Animals}

In total, 30 client-owned dogs were included in the study. All dogs were inpatients at the Department of Internal Medicine at the Clinic of Dog and Cat Diseases at the University of Veterinary and Pharmaceutical Sciences, Brno, Czech Republic. The average age and weight of dogs was 6 years and $27.1 \mathrm{~kg}$; sixteen were males and 14 females. There were 5 mongrel dogs, 3 Rhodesian Ridgebacks, 2 Labrador Retrievers, 2 Hungarian Vizsla, and one each of other breeds (Dachshund, West Highland White Terrier, Petit Basset Griffon Vendéen, French Bulldog, Rottweiler, Akita, Bernese Mountain Dog, Miniature Pinscher, Golden Retriever, Cane Corso, English Cocker Spaniel, German Shepherd Dog, Hovawart, Beauceron, Yorkshire Terrier and Australian Shepherd).

The most common cause of dog hospitalization was gastrointestinal disease (haemorrhagic/bacterial gastroenteritis and pancreatitis), followed by haemato-oncological (cancer, haemolytic anaemia), respiratory (bacterial pneumonia and abscesses, eosinophilic bronchopneumopathy) and genito-urinary disorders (pyometra and prostatitis). The dogs were hospitalized due to various conditions that required the control of a primary bacterial disease (i.e. pyometra) or prevention of a secondary bacterial infection, none of which were lifethreatening.

\section{Study design}

The dogs were randomly divided into 2 groups of 15 . The control group received amoxicillin-clavulanate (Augmentin ${ }^{2}$, GlaxoSmithkline, UK) at a dose of $22 \mathrm{mg} / \mathrm{kg}$ intravenously (i.v.), $3 \times$ daily; the experimental group received enrofloxacin (Enroxil ${ }^{\circledR}, \mathrm{Krka}$, Slovenia) at a dose of $5 \mathrm{mg} / \mathrm{kg}$ subcutaneously (s.c.), $2 \times$ daily in combination with amoxicillin-clavulanate $(22 \mathrm{mg} / \mathrm{kg}$ i.v.). Concurrently, both groups received other drugs such as ranitidine and maropitant which are not reported to provoke any ECG (QTi) changes and are not known to have cardioprotective effects. None of the dogs had a history or any current heart disease that could also affect the QTi. All dogs were examined daily and underwent complete haematology and biochemistry examinations to rule out other factors that could influence the QTi.

The study was designed to be a single-blinded randomized controlled clinical trial. Ethical approval was given by the Animal Care and Use Committee of the University of Veterinary and Pharmaceutical Sciences, Brno, Czech Republic.

\section{Electrocardiographic measurements}

Electrocardiograms of dogs in a standing position were recorded using a digital electrocardiograph (Cardy $($ ) Pet, Hungary) once-a-day, starting at day 0 (before any intervention) until the $5^{\text {th }}$ day of hospitalization using the same time window (11-13 h). A period of adaptation for the dogs was given to allow the HR to stabilize. Standard limb leads were used for recording 30 s ECG segments. No electronic filters were used during ECG signal acquisition. The ECGs were recorded at $50 \mathrm{~mm} / \mathrm{s}$ on a chart recorder calibrated to display signals at $1 \mathrm{~cm} /$ $\mathrm{mV}$. Measurements of HR, P wave (width and height), PQ, QRS (Q wave, R wave, S wave and width), QT, ST and $T$ waves (positive and negative component) were carried out automatically using appropriate software, and then manually. For each parameter, a value of 12 consecutive heart beats was used in the analysis, which corresponded to approximately 3 respiratory cycles to minimize the effects of sinus arrhythmia (SA). 
Table 2. Mathematical formulae to correct for heart rate-induced changes in the QT interval of the electrocardiogram.

\begin{tabular}{ll}
\hline Formula & Reference \\
\hline QT ms $/ \sqrt{ }$ RR s & Bazzet 1920 \\
QT ms $/ \sqrt{ }$ RR s & Fridericia 1920 \\
QT ms $-0.087($ RR ms -1000$)$ & Van de Water 1989 \\
QT ms $+0.154(1-$ RR s) & Sagie et al. (the Framingham study) 1992 \\
\hline
\end{tabular}

$\mathrm{ms}$ - milliseconds, $\mathrm{s}$ - seconds, $\mathrm{RR}$ - electrocardiographic interval between $2 \mathrm{R}$ waves)

QT analysis

QTi was defined from the earliest onset of the QRS complex to the latest end of the T wave, which was determined from a straight line extrapolation of the terminal portion of the $\mathrm{T}$ wave to the point where it intersects the baseline according to recommendations of Friedman et al. (2007). One investigator developed inclusion criteria and data acquisition, while another investigator blindly analyzed the ECGs. As HR can be influenced by both internal as well as external factors, such as drugs, the QTi was corrected (corrected QT interval: QTc) using four different classical formulae: Bazzet, Fridericia, Van de Water and Framingham (Table 2). A drug-induced effect on the QTi was defined as the difference in change in QTi or QTc.

Statistical analysis

The results were expressed as means \pm SD. Normality of variables was tested with the Shapiro-Wilk test. Student's unpaired $t$-test was used to compare QTi and QTc between groups. Changes in the ECG parameters in each group during the study were determined by analysis of variance for repeated measures. All tests were twotailed and carried out at the 0.05 level of significance.

\section{Results}

Comparison of the two groups over a period of 5 consecutive days showed no significant differences $(P>0.05)$ in QTi and QTc or in other ECG parameters (Table 3).

No adverse reactions were reported in both groups of dogs and no arrhythmias or changes in blood pressure were detected.

\section{Discussion}

Prolongation of QT is generally categorized into primary (inherited, familial, congenital, idiopathic) and secondary (disease, drug or toxin induced) (Moss 1999; Wang et al. 2003; Gupta et al. 2007). The pharmacological mechanism for QTi prolongation occurs as some drugs can cause effects that resemble the mutant gene hERG on chromosome 7 that encodes an abnormal $\mathrm{K}^{+}$channel protein, thus blocking cardiac voltage-gated potassium channels (IK $)$ (Friedman et al. 2007). Amoxicillin-clavulanate was selected for the control group of dogs as it is the most commonly used antibiotic in our practice and there are no reports describing an effect on the QTi. On the other hand, quinolones can prolong QTi and are directly correlated to the risk of developing ventricular tachyarrhythmic episodes (Moss 1999).

No reports studying the effects of ciprofloxacin in dogs showed any effects on the ECG. Toyoshima et al. (2005) used parenteral ciprofloxacin (5-200 mg/kg) and confirmed that this drug is a so called "negative compound" for the QTi. Another study used a continual infusion of ciprofloxacin which produced a significant decrease in the HR with concurrent prolongation of the QTi (Ghaffari and Parsamehr 2009). The authors concluded that the reduction in HR, and not the drug, was the reason for this minimal QTi prolongation. In contrast, another study reported mild increases in the HR ( $\mathrm{Lu}$ et al. 2008). It is possible that these differing reports on the HR are the result of other factors such as circadian cycle, manipulation, age, sex and others. 
Table 3. Electrocardiographic values of the QTi and QTc in hospitalized dogs treated with amoxicillin-clavulanate and in combination with enrofloxacin in a 5 days interval.

\begin{tabular}{|c|c|c|c|c|c|}
\hline & & & Day 0 & & \\
\hline QT interval (ms) & QT & QTc(b) & QTc(f) & QTc(vw) & QTc(fr) \\
\hline Control & 218.6 & 249.34 & 232.28 & 256.41 & 219.02 \\
\hline Enrofloxacin & 209.6 & 241.81 & 233.45 & 238.69 & 208.64 \\
\hline \multirow[t]{2}{*}{$P$ value } & 0.28 & 0.11 & 0.2 & 0.29 & 0.35 \\
\hline & \multicolumn{5}{|c|}{ Day 1} \\
\hline QT interval (ms) & QT & QTc(b) & QTc(f) & QTe(vw) & QTc(fr) \\
\hline Control & 210.6 & 276.32 & 251.9 & 297.61 & 210.72 \\
\hline Enrofloxacin & 203 & 265.81 & 242.7 & 290.01 & 203.12 \\
\hline \multirow[t]{2}{*}{$P$ value } & 0.29 & 0.22 & 0.14 & 0.29 & 0.29 \\
\hline & \multicolumn{5}{|c|}{ Day 2} \\
\hline QT interval (ms) & QT & QTc(b) & QTc(f) & QTc(vw) & QTc(fr) \\
\hline Control & 211.2 & 268.51 & 247.14 & 298.14 & 211.25 \\
\hline Enrofloxacin & 204.73 & 271.36 & 246.5 & 291.68 & 204.79 \\
\hline \multirow[t]{2}{*}{$P$ value } & 0.39 & 0.77 & 0.92 & 0.39 & 0.39 \\
\hline & \multicolumn{5}{|c|}{ Day 3} \\
\hline QT interval (ms) & QT & QTc(b) & QTc(f) & QTe(vw) & QTe(fr) \\
\hline Control & 209.86 & 283.29 & 256 & 296.81 & 209.93 \\
\hline Enrofloxacin & 208.2 & 272.18 & 248.36 & 295.14 & 208.26 \\
\hline \multirow[t]{2}{*}{$\mathrm{P}$ value } & 0.81 & 0.26 & 0.29 & 0.818 & 0.81 \\
\hline & \multicolumn{5}{|c|}{ Day 4} \\
\hline QT interval (ms) & QT & QTc(b) & QTc(f) & QTc(vw) & QTc(fr) \\
\hline Control & 214.26 & 281.01 & 256.27 & 301.21 & 214.32 \\
\hline Enrofloxacin & 204.26 & 265.4 & 242.72 & 291.21 & 204.32 \\
\hline \multirow[t]{2}{*}{$P$ value } & 0.18 & 0.14 & 0.08 & 0.18 & 0.18 \\
\hline & \multicolumn{5}{|c|}{ Day 5} \\
\hline QT interval (ms) & QT & QTc(b) & QTc(f) & QTc(vw) & QTc(fr) \\
\hline Control & 213.2 & 291.91 & 262.05 & 300.15 & 213.26 \\
\hline Enrofloxacin & 202,9 & 272.14 & 247.17 & 291.54 & 204.66 \\
\hline$P$ value & 0.23 & 0.08 & 0.06 & 0.23 & 0.23 \\
\hline
\end{tabular}

QTc(b) (Bazzet), QTc(f) (Fridericia), QTc(vw) (Van der Water) and QTc(fr) (Framingham)

In our study, we did not show any effects on the QTi and QTc or in other ECG parameters during the 5-day treatment with enrofloxacin in hospitalized dogs. In recent scientific literature, a number of studies concluded that certain quinolones have no effect on the QTi. In humans, treatment with ciprofloxacin sporadically caused prolongation of the QTi and arrhythmia, and when present, was associated with other drugs that potentially prolonged repolarization (Flanagan et. al 2006). Since ciprofloxacin is a metabolite of enrofloxacin, we could deduce that the absence of changes in QTi and QTc are mainly due to its chemical characteristics, which are very similar for both antibiotics. Furthermore, the dogs in our study did not have any other condition that has been reported to prolong the QTi. Hypokalaemia, hypomagnesaemia, left ventricular hypertrophy and congestive heart failure are conditions associated with abnormalities of ventricular repolarization and QTi prolongation in human beings (Moss 1999) as well as in dogs (Koyama et al. 2004).

From our experience, we prefer subcutaneous administration of enrofloxacin as we 
have observed episodes of collapse after rapid intravenous administration in clinical practice. These signs have been explained as hypotension, due in part to the release of histamine (Chanoit et al. 2005). In the dogs in this study, we did not observe any clinical signs suggesting hypotension, although we did not carry out continual blood pressure monitoring. A 5-day study period was selected, since it represents average hospitalization time in veterinary practice. We assumed that the specific time when the ECGs were carried out in this study represented the time when the plasma drug concentrations reached a peak. Also, our methodology seems more robust than the reported small sample size (usually less than $10 \mathrm{dogs}$ ), single-dose, and single agent studies that have shown conflicting results with respect to QTc prolongation and may not describe the effects seen with repeated-dose administration (Shah 2002). Dogs are prone to various forms of SA; therefore the QTi was corrected by the most accepted QTc approaches. We used four classical QTc formulae which have all been used in dogs before and have shown specific advantages for this study.

We conclude that the parenteral use of enrofloxacin is safe and effective and does not produce effects on the QTi that could lead to ventricular arrhythmias. We cannot conclude that the same level of safety is present also in dogs that have other factors that influence QTi (i.e. cardiac disease). Further in-depth studies evaluating the influence of such preparations on atrial and ventricular repolarization should be conducted.

\section{Acknowledgments}

The research was funded by the Internal Grant Agency of the University of Veterinary and Pharmaceutical Sciences Brno (IGA-VFU, project no. 256/2009/FVL). The authors would like to thank Dr. Shachar Dvir, DVM for help with the revision of the English text.

\section{References}

Bazett HC 1920: An analysis of the time relationship of electrocardiograms. Heart 7: 353-370

Boothe DM, Boeckh A, Boothe HW, Wilkie S 2001: Tissue concentrations of enrofloxacin and ciprofloxacin in anesthetized dogs following single intravenous administration. Vet Therapeutics 2: 120-128

Chanoit GP, Schneider M, Woehrlé F, Lefebvre HP 2005: Effect of marbofloxacin on cardiovascular variables in healthy isoflurane-anesthetized dogs. Am J Vet Res 66: 2090-2094

Dennis SG, Wotton PR, Boswood A, Flaherty D 2007: Comparison of the effects of thiopentone and propofol on the electrocardiogram of dogs. Vet Rec 160: 681-686

Fenichel RR, Malik M, Antzelevitch C, Sanguinetti M, Roden DM, Priori SG, Ruskin JN, Flanagan MC, Mitchell ES, Haigney MC 2006: Ciprofloxacin-induced torsade de pointes. Int J Cardiol 113: 239-241

Fridericia LS 1920: The duration of systole in the electrocardiogram of normal subjects and of patients with heart disease. Acta Med Scand 53: 469-486

Friedman MJ, Mull CC, Sharieff GQ, Tsarouhas N 2003: Prolonged QT syndrome in children: an uncommon but potentially fatal entity. J Emerg Med 24: 173-179

Ghaffari MS, Parsamehr R 2009: The effects of intravenous ciprofloxacin on the electrocardiogram of healthy dogs. Vet Res Commun 33: 987-990

Gupta A, Lawrence AT, Krishnan K, Kavinsky CJ, Trohman RG 2007: Current concepts in the mechanisms and management of drug-induced QT prolongation and torsade de pointes. Am Heart J 153: 891-899

Koyama H, Yoshii H, Yabu H, Kumada H, Fukuda K, Mitani S, Rousselot JF, Virose H, Uchino T 2004: Evaluation of QT interval prolongation in dogs with heart failure. J Vet Med Sci 66: 1107-1111

Lu GC, She JH, Jiang H, Li YZ, Yuan BJ 2008: Sixty-day repeated dose toxicity of sinafloxacin in rats and dogs. Food Chem Toxicol 46: 575-580

Moss AJ 1999: The QT interval and torsade de pointes. Drug Saf 21: 5-10

Sagie A, Larson MG, Goldberg RJ, Bengston JR, Levy D 1992: An improved method for adjusting the QT interval for heart rate (the Framingham Heart Study). Am J Cardiol 70: 797-801

Shah RR 2002: The significance of QT interval in drug development. Br J Clin Pharmacol 54: 188-202

Sheridan DJ 2000: Drug-induced proarrhythmic effects: assessment of changes in QT interval. Br J Clin Pharmacol 50: 297-302

Toyoshima S, Kanno A, Kitayama T, Sekiya K, Nakai K, Haruna M, Mino T, Miyazaki H, Yano K, Yamamoto k 2005: QT PRODACT: in vivo QT assay in the conscious dog for assessing the potential for QT interval prolongation by human pharmaceuticals. J Pharmacol Sci 99: 459-471 
Van de Water A, Verheyen J, Xhonneux R, Reneman RS 1989: An improved method to correct the QT interval of the electrocardiogram for changes in heart rate. J Pharmacol Methods 22: 207-217

Wang J, Della Penna K, Wang H, Karczewski J, Connolly TM, Koblan TS, Bennett PB, Salata JJ 2003: Functional and pharmacological properties of canine ERG potassium channels. Am J Physiol Heart Circ Physiol 284: 256-267 